\title{
THE BEHNKE-STEIN THEOREM FOR OPEN RIEMANN SURFACES
}

\author{
R. R. SIMHA
}

(Communicated by Irwin Kra)

\begin{abstract}
Using the Riemann-Roch theorem and the set-topological part of Bishop's special polyhedron lemma, we show that the usual Runge approximation theorem for compact subsets of the Riemann sphere is valid word-for-word on any compact Riemann surface $X$, with meromorphic functions on $X$ playing the role of rational functions; this result is essentially equivalent to the Behnke-Stein approximation theorem.
\end{abstract}

The Behnke-Stein generalization of the Runge approximation theorem [1], which is the basic tool for many existence questions on open Riemann surfaces, can be stated in several equivalent ways, for instance:

Let $X$ be a Riemann surface, and $K$ a compact subset of $X$. Then, in order that every holomorphic function in a neighborhood of $K$ be uniformly approximable on $K$ by holomorphic functions on $X$, it is necessary and sufficient that $X-K$ have no connected component with compact closure in $X$.

As is well known, the necessity part of the above theorem follows from the sufficiency part (using the theory of compact Riemann surfaces). In this note, we wish to point out how the famous "special polyhedron lemma" of Bishop [2], which is an elementary set-topological result, can be used (together with the Riemann-Roch theorem for compact Riemann surfaces) to give a simple proof of the following theorem.

Theorem 1.1. Let $X$ be a compact Riemann surface, and $K \subset X$ a compact subset. Let $Q$ be any subset of $X-K$ which contains (precisely) one point $q_{i}$ from each connected component $W_{i}$ of $X-K$. Then any holomorphic function on a neighborhood of $K$ can be approximated uniformly on $K$ by meromorphic functions on $X$ whose poles lie in $Q$.

Again it is well known and easy to see that Theorem 1.1 implies the sufficiency part of Behnke-Stein. The main fact needed for this implication is the following:

Received by the editors March 24, 1988.

1980 Mathematics Subject Classification (1985 Revision). Primary 30E10, 30F10.

Key words and phrases. Riemann surfaces, Behnke-Stein approximation. 
any relatively compact open subset of any Riemann surface can also be regarded as an open subset of a compact Riemann surface. Observe that, when $X=\mathbb{C} \cup \infty$ is the Riemann sphere and $K \subset \mathbb{C}$, and we take $q_{\infty}=\infty$ for the component $W_{\infty}$ of $X-K$ containing $\infty$, Theorem 1.1 reduces to the usual Runge theorem in $\mathbb{C}([3]$, p. 176).

The proof of Theorem 1.1 is given in $\S 3$; the preliminaries needed, which are easy consequences of the Riemann-Roch theorem, are proved in $\S 2$. In $\S 4$, we state Bishop's lemma precisely, and indicate how it is especially easy to apply it in our case.

\section{Preliminaries}

Notation 2.1. For any open subset $U$ of the compact Riemann surface $X, O(U)$ (resp. $M(U)$ ) denotes the set of holomorphic (resp. meromorphic) functions on $U$. For any $A \subset X, M_{A}(X)$ denotes the set of $f \in M(X)$ whose poles lie in $A$.

For any divisor $D=\sum n_{i} p_{i}$ on $X$ (finite sum with the $p_{i}$ distinct), $L(D)$ denotes the sheaf of germs of holomorphic sections of the line bundle $L(D)$ corresponding to $D$. For any open $U \subset X$, we make the usual identification $H^{0}(U, L(D))=\left\{f \in M(U): f\right.$ has a zero (resp. pole) of order $\geq-n_{i}$ (resp. $\leq n_{i}$ ) at each $p_{i} \in U$ with $n_{i}<0$ (resp. $\left.\left.>0\right)\right\}$. The $H^{0}(U, L(D))$ are Frechet spaces in the topology of uniform convergence on compact subsets of $U$, e.g. they can be regarded as closed subspaces of $O(U-\operatorname{Supp}(D)), \operatorname{Supp}(D)=$ $\left\{p_{1}, p_{2}, \ldots\right\}$ :

We shall use the Riemann-Roch theorem for compact Riemann surfaces in the following form.

There exists an integer $\delta(X)=\delta$ such that, for any divisor $D=\sum n_{i} p_{i}$ on $X$ with $\operatorname{deg}(D):=\sum n_{i} \geq \delta, H^{1}(X, L(D))=0$. (As is well known, we can take $\delta=2 g-1$, where $g$ is the genus of $X$.)

Lemma 2.2. Fix $q_{0} \in X$ arbitrarily. Let $p_{1}, \ldots, p_{n}$ be distinct points in $X-q_{0}$, and $\left(U_{i}, z_{i}\right)$ coordinate charts at the $p_{i}$. For $1 \leq i \leq n$, let $l_{i}$ be Laurent polynomials: $l_{i}(T)=\sum_{j=-d_{i}}^{d_{i}} a_{i j} T^{j} \in \mathbb{C}\left[T, T^{-1}\right], d_{i}$ integers $\geq 0$. Then there exists $f \in M(X)$, with poles at the most at $q_{0}, p_{1}, \ldots, p_{n}$, such that, for each $i, f-l_{i}\left(Z_{i}\right)$ has a zero of order $\geq d_{i}+1$ at $p_{i}$.

Proof. Let $D$ be the divisor $d_{0} q_{0}-\sum\left(d_{i}+1\right) p_{i}$, with $d_{0}$ so large that $\operatorname{deg}(D) \geq$ $\delta$. We may suppose that the $U_{i}$ are pairwise disjoint, and regard the $l_{i}\left(Z_{i}\right)$ as defining a 1-cocycle for $L(D)$ with respect to the covering $\left(U_{1}, \ldots, U_{n}, X-\right.$ $\left.\left\{p_{1}, \ldots, p_{n}\right\}\right)$. This cocycle is a coboundary by Riemann-Roch, and the lemma follows from this.

Corollary 2.3. For every $p \in X-q_{0}$, there exists $f_{p} \in M_{p, q_{0}}(X)$ with a simple pole at $p$.

Lemma 2.4. Let $p, f_{p}$ be as in 2.3. Let $\alpha: I(=[0,1] \subset \mathbf{R}) \rightarrow X-q_{0}$ be a path in $X-q_{0}$. Then there is a continuous map $t \rightarrow f_{t}$ of $I$ into $M(X)$ such that 
(i) $f_{0}=f_{p}$, and (ii) $f_{t} \in M_{\alpha(t), q_{0}}$ and has a simple pole at $\alpha(t)$, for all $t \in I$. (The continuity of $t \rightarrow f_{t}$ means that the map $(t, x) \rightarrow f_{t}(x)$ of $I \times X$ into $\mathbb{C} \cup \infty$ is continuous.)

Proof. Clearly, we may assume that there is a coordinate disc $(U, z)$ with $q_{0} \notin U$ and $\alpha(I) \subset U$. Let $V \subset \subset U$ be a smaller disc with $\alpha(I) \subset V$. There is a $\lambda \in \mathbb{C}^{*}$ such that $f_{p}-\lambda /(z-z(p))$ is holomorphic in $U$. Put $\beta(t)=z(\alpha(t))$, and regard $t \rightarrow l_{t}=\lambda(z-\beta(t))^{-1}-\lambda(z-\beta(0))^{-1}$ as a continuous map $I \rightarrow H^{0}\left(U-\bar{V}, L\left(\delta q_{0}\right)\right)=O(U-\bar{V})$. By Riemann-Roch, the obvious sequence

$$
0 \rightarrow H^{0}\left(X, L\left(\delta q_{0}\right)\right) \rightarrow H^{0}\left(X-\bar{V}, L\left(\delta q_{0}\right)\right) \oplus O(U) \rightarrow O(U-\bar{V}) \rightarrow 0
$$

is exact. Moreover, $H^{0}\left(X, L\left(\delta q_{0}\right)\right)$ is finite dimensional, hence this sequence, regarded as an exact sequence of continuous linear maps of Frechet spaces, splits. (An explicit splitting is easy to give in this special case.) Hence we have continuous maps $t \rightarrow \varphi_{t}, t \rightarrow \psi_{t}$ of $I$ into $H^{0}\left(X-\bar{V}, L\left(\delta q_{0}\right)\right)$ and $O(U)$ respectively, such that $\varphi_{0}=\psi_{0}=0$ and $\varphi_{t}-\psi_{t}=l_{t}$ on $U-\bar{V}$. Setting $g_{t}=\varphi_{t}$ on $X-\bar{V}$ and $l_{t}+\psi_{t}$ on $U$, we see that the family $t \rightarrow f_{p}+g_{t}, t \in I$ has the desired properties.

Corollary 2.5. Let $\alpha: I \rightarrow X$ be a path with $\alpha(0)=p\left(\neq q_{0}\right)$ and $\alpha(1)=q_{0}$. Let $K \subset X$ be a compact set with $K \cap \alpha(I)=\varnothing$. Then every $f \in M_{p, q_{0}}(X)$ can be approximated uniformly on $K$ by elements of $M_{q_{0}}(X)$.

Proof. We may assume $\alpha(t) \neq q_{0}$ for $t<1$. Pick (e.g. using Lemma 2.2) any nonconstant $f_{q_{0}} \in M_{q_{0}}(X)$. Let $V$ be a neighborhood of $q_{0}$ such that $\inf \left\{\left|f_{q_{0}}(x)\right|: x \in V\right\}>2 \sup \left\{\left|f_{q_{0}}(x)\right|: x \in K\right\}$. Choose $t^{\prime} \in I$ such that $\alpha\left(t^{\prime}\right) \in$ $V$. Let $\left(f_{t}\right)$ be the functions constructed in 2.4 for the path $\alpha\left[0, t^{\prime}\right]$. Clearly, we can subdivide $\left[0, t^{\prime}\right]: 0=t_{0}<t_{1}<\cdots<t_{n}=t^{\prime}$ such that $\left|f_{t_{i}}\left(\alpha\left(t_{i-1}\right)\right)\right|>$ $2 M, i=1, \ldots, n$, where $M:=\sup \left\{\left|f_{t}(x)\right|: 0 \leq t \leq t^{\prime}, x \in K\right\}(<\infty)$.

Now let $f \in M_{p, q_{0}}(X)$. Then $f\left(f_{t_{1}}-f_{t_{1}}(p)\right)^{m} \in M_{\alpha\left(t_{1}\right), q_{0}}(X)$ for some integer $m>0$. And $\left(f_{t_{1}}(p)-f_{t_{1}}\right)^{-1}=f_{t_{1}}(p)^{-1}\left(1-f_{t_{1}} / f_{t_{1}}(p)\right)^{-1}$ can be approximated on $K$ by a polynomial in $f_{t_{1}}$ using the geometric series. Repeating this argument $n-1$ more times, we see that $f$ can be approximated on $K$ by elements of $M_{\alpha\left(t^{\prime}\right), q_{0}}(X)$. The same argument, repeated this time using $f_{q_{0}}$, shows that any $g \in M_{\left(\alpha\left(t^{\prime}\right), q_{0}\right.}(X)$ can be approximated on $K$ by elements of $M_{q_{0}}(X)$, and the corollary is proved.

We now come to the proof of Theorem 1.1. With the notation of 1.1, let $U \supset K$ be an open set. For each $p \in \partial U$, there exists $f_{p} \in M_{p}(X)$ with a pole at $p$. We may assume that $\left|f_{p}\right|<1$ on $K$. Let $V_{p}$ be a neighborhood of $p$ such that $\left|f_{p}\right|>2$ on $V_{p}$. Finitely many of the $V_{p}$, say $V_{p_{1}}, \ldots, V_{p_{m}}$, cover 
$\partial U$. Thus, with

$$
V:=\left\{x \in U:\left|f_{p_{i}}(x)\right|<1 \text { for } 1-\leq i \leq m\right\}
$$

we have $K \subset V \subset \subset U$. By the procedure due to Bishop [2] (see $\S 4$ ), we can then find a $\varphi \in M(X)$ which is in fact a polynomial in the $f_{p_{i}}$, and an open set $V_{0} \subset V$, such that $K \subset V_{0}$, and $\varphi: V_{0} \rightarrow \Delta(=\{|z|<1\} \subset \mathbb{C}\}$ is a proper map. Hence it is clear that the following lemma, together with Corollary 2.5, implies Theorem 1.1.

Lemma 3.1. Let $V_{0}$ be any open subset of $X$, and $\varphi: V_{0} \rightarrow \Delta$ a proper holomorphic map to the unit disc in $\mathbb{C}$. Let $q_{0} \in X-V_{0}$ be arbitrary. Then any $h \in O\left(V_{0}\right)$ can be approximated uniformly on any compact subset $K$ of $V_{0}$ by polynomials in $\varphi$ and the elements of $M_{q_{0}}(X)$.

Proof. Since $\varphi: V_{0} \rightarrow \Delta$ is a proper map, there exists a discrete subset $E$ of $\Delta$ such that $\varphi: V_{0}-\varphi^{-1}(E) \rightarrow \Delta-E$ is a (possibly disconnected) finite-sheeted (say $n$-sheeted) covering. Pick some $z_{0} \in \Delta-E$ : by Lemma 2.2, there exists $\varphi_{1} \in M_{q_{0}}(X)$ taking $n$ distinct values on $\varphi^{-1}\left(Z_{0}\right)$. Let $P(z, T) \in O(\Delta)[T]$ be the monic polynomial of degree $n$, defined for $z \in \Delta-E$ by $P(z, T)=$ $\prod_{\varphi(p)=z}\left(T-\varphi_{1}(p)\right)$. Define $\psi \in O\left(V_{0}\right)$ by $\psi(p)=(\partial P / \partial T)\left(\varphi(p), \varphi_{1}(p)\right)$. Then $\psi \neq \equiv 0$ on any connected component of $V_{0}$, since $\psi \neq 0$ on $\varphi^{-1}\left(z_{0}\right)$.

Now, for any disc $\Delta_{1} \subset \Delta$, and $V_{1}:=\varphi^{-1}\left(\Delta_{1}\right)$, consider, for any $h \in O\left(V_{1}\right)$, the polynomial $Q_{h} \in O(\Delta)[T]$, defined for $z \in \Delta_{1}-E$ by

$$
Q_{h}(z, T)=\sum_{i=1}^{n} h\left(p_{i}\right) \prod_{j \neq i}\left(T-\varphi_{1}\left(p_{j}\right)\right),
$$

where $\left\{p_{1}, \ldots, p_{n}\right\}=\varphi^{-1}(z)$. Then $Q_{h}\left(\varphi(p), \varphi_{1}(p)\right)=h(p) \psi(p)$ for $p \in$ $V_{1}-\varphi^{-1}(E)$ by definition, hence for all $p \in V_{1}$.

Now we can choose $\Delta_{1} \subset \subset \Delta$ such that $\varphi(K) \subset \Delta_{1}$. Then $V_{1} \subset \subset V_{0}$, hence $\psi$ has only finitely many zeros in $V_{1}$. Hence, for any given $h \in O\left(V_{1}\right)$, we can find (by Lemma 2.2) a $\varphi_{2} \in M_{q_{0}}(X)$ such that $h-\varphi_{2}$ is divisible by $\psi$ in $O\left(V_{1}\right)$ : say $h-\varphi_{2}=\psi h^{\prime}$. But $\psi\left(p^{\prime}\right) h^{\prime}(p)=Q_{h^{\prime}}\left(\varphi(p), \varphi_{1}(p)\right)$ for the $Q_{h^{\prime}} \in$ $O\left(\Delta_{1}\right)[T]$ constructed above. Thus, using Taylor expansions to approximate the coefficients of $Q_{h^{\prime}}$ on $\varphi(K)$, we see that $\psi h$ can be approximated on $K \Subset \varphi^{-1}(\varphi(K))$ by polynomials in $\varphi$ and $\varphi_{1}$.

\section{BISHOP's LEMMA}

We state below the lemma of Bishop used in $\S 3$. Since we have not found a reference where the result is proved in the stated form, we first state the two simple facts using which the lemma can be easily proved. 
Lemma 4.1 ([2], p. 222). For $\nu \geq 1$, the set $\left\{z \in \mathbb{C}:\left|z^{\nu}-1\right|<\frac{1}{2}\right\}$ is contained in the union of the $\nu$ pairwise disjoint sets

$W_{k, \nu}=\left\{\left(\frac{1}{2}\right)^{1 / \nu}<|z|<\left(\frac{3}{2}\right)^{1 / \nu},|\arg z-2 k \pi / \nu|<\pi / \nu\right\}, k=0, \ldots, \nu-1$;

the diameter of $W_{k, \nu}$ is $O\left(\nu^{-1}\right)$ as $\nu \rightarrow \infty$.

Lemma 4.2. Let $Y, Z$ be metric spaces, with $Y$ compact, and $f: Y \rightarrow Z$ a continuous map with totally disconnected fibers. Then, for every $\varepsilon>0$, there exists $\delta>0$ such that every connected subset of $Y$ of diameter $\geq \varepsilon$ has $f$-image of diameter $\geq \delta$.

The argument of Bishop [2] for his proof of Theorem 2 (p. 221 of [2]) proves exactly the following Lemma 4.3: 4.2 can be used in place of the function theory on p. 223 of [2].

Bishop's Lemma 4.3. Let $U$ be a locally compact locally arcwise-connected metric space, and $f_{i}: U \rightarrow \mathbb{C}$ continuous functions $1 \leq i \leq m$. Let $V \subset \subset U$ be an open subset such that $f=\left(f_{1}, \ldots, f_{m}\right)$ maps $V$ properly into the unit polydisc $\left\{Z_{i}<1,1 \leq i \leq m\right\}$ in $\mathbb{C}^{m}$. Let $U_{1}=\left\{x \in U: f_{1}(X) \neq 0\right\}$, and suppose the map $g=\left(f_{2} / f_{1}, \ldots, f_{m} / f_{1}\right): U_{1} \rightarrow \mathbb{C}^{m-1}$ has totally disconnected fibers. Then, for any compact $K \subset V$, there exist $r>1$ and a positive integer $\nu$ such that the set

$$
V^{\prime}=\left\{x \in U:\left|\left(r f_{1}(x)\right)^{\nu}-\left(r f_{j}(x)\right)^{\nu}\right|<1, j=2, \ldots, m\right\}
$$

contains $K$, and the union $V_{0}$ of the connected components of $V^{\prime}$ meeting $K$ is contained in $V$. Hence $V_{0} \subset \subset U$, and the $m-1$ functions $\left(r f_{1}\right)^{\nu}-\left(r f_{j}\right)^{\nu}, j=$ $2, \ldots, m$ map $V_{0}$ properly into the unit polydisc in $\mathbb{C}^{m-1}$.

We conclude with the observation that, in our applications of this lemma, the condition that the map $g: U_{1} \rightarrow \mathbb{C}^{m-1}$ have discrete fibers is automatically satisfied. This is because the $f_{i}$ will be holomorphic functions on a connected Riemann surface $X^{\prime}$ containing $U$ as an open set. Thus $g$ will fail to satisfy the required condition only if $g_{j}=\lambda_{j} g_{1}, \lambda_{j} \in \mathbb{C}, j \geq 2$. In this case, it is obvious that a suitable constant multiple $\varphi:=\lambda g_{1}$ will already have the desired property that $\varphi: V \rightarrow \Delta$ be a proper map. Thus, after at the most $m-1$ applications of Bishop's lemma, we will have $V_{0}$ and $\varphi$ as required in $\S 3$.

\section{REFERENCES}

1. H. Behnke and K. Stein, Entwicklungen analytischer Funktionen auf Riemannschen Flächen, Math. Ann. 120 (1948), 430-461.

2. E. Bishop, Mappings of partially analytic spaces, Amer. J. Math. 83 (1961), 209-242.

3. S. Saks and A. Zygmund, Analytic functions, Warsaw, 1952.

School of Mathematics, Tata Institute of Fundamental Research, Homi Bhabha ROAD, BOMBAY 400 005, INDIA 